\title{
Linguistic Cues to Deception Assessed by Computer Programs: A Meta-Analysis
}

\author{
Valerie Hauch, \\ Justus-Liebig-University of Giessen, \\ Germany \\ Valerie.Hauch@psychol .uni- \\ giessen.de \\ Jaume Masip, \& \\ University of Salamanca, \\ Spain \\ jmasip@usal.es
}

\begin{abstract}
Research syntheses suggest that verbal cues are more diagnostic of deception than other cues. Recently, to avoid human judgmental biases, researchers have sought to find faster and more reliable methods to perform automatic content analyses of statements. However, diversity of methods and inconsistent findings do not present a clear picture of effectiveness. We integrate and statistically synthesize this literature. Our meta-analyses revealed small, but significant effect-sizes on some linguistic categories. Liars use fewer exclusive words, self- and other-references, fewer time-related, but more space-related, negative and positive emotion words, and more motion verbs or negations than truth-tellers.
\end{abstract}

\section{Introduction}

Meta-analytic findings indicate that human judges are just slightly better than chance at discriminating between truths and lies (Bond, \& DePaulo, 2006). Likewise, meta-analyses of training programs designed to teach lie detection have shown a small to medium effect size in improving judges' detection accuracy (e.g., Hauch, Sporer, Michael, \& Meissner, 2010). Together, these findings suggest that there is a great need to better understand factors involved in deception and find ways to improve its detection. Attempts at these tasks have led researchers to use computer programs to analyze

\author{
Iris Blandón-Gitlin, \\ California State University, Fullerton, \\ USA \\ iblandon-gitline.fullerton.edu \\ Siegfried L. Sporer \\ Justus-Liebig-University of Giessen, \\ Germany \\ Sporerapsychol.uni-giessen.de
}

linguistic markers in truthful and deceptive statements. A number of verbal cues have been shown to differ in lies and truths (DePaulo, Lindsay, Malone, Muhlenbruck, Charlton, \& Cooper, 2003; Sporer, 2004, Vrij, 2008), and teaching content cues has shown to improve detection more effectively than teaching nonverbal or paraverbal cues (Hauch et al., 2010).

The automatization of lie detection is appealing for at least two reasons. First, such systems can be considered more objective than human judges who are prone to biases (Levine, Park, \& McCornack, 1999). Second, online judgments of various deception cues from videos or transcripts can tax the cognitive capacity of judges and lead to time delays and errors. Researchers have used different computer programs for the evaluation of the truth status. Computers can quickly analyze large amounts of text and provide more reliable data. Moreover, the linguistic categories evaluated across studies have varied. In some cases, the direction of the effect for the same linguistic categories has been opposite across studies, or opposite to theoretically-based predictions.

These methodological differences and inconsistencies in findings calls for a quantitative analysis and integration of findings. This is the goal of the present meta-analytic review.

\section{Method}

After a thorough literature search (Social Sciences Citation Index, PsycInfo, Dissertation Abstracts, Google Scholar, and cited reference searches), a large number $(k=84)$ of published 
and unpublished studies were located. Studies were only included into the meta-analysis if they meet several inclusion criteria.

\subsection{Inclusion Criteria}

- Use of computer-based method/program to analyze transcripts in terms of specific linguistic categories;

- Datasets of transcripts (from spoken or written language) which include deceptive and truthful accounts;

- Independence of datasets;

- Specific linguistic categories applied to predict truth status;

- Sufficient statistical data (means and standard deviation separately for lies and truths) to calculate effect sizes (Cohen's d) for specific categories;

- Sources written in English, Spanish, or German.

\subsection{Exclusion Criteria}

- Psychophysiological methods or use of subjective ratings;

- Ground truth of real statements only established from verdicts or media commentaries (or not established);

- Only computer-analysed linguistic variable is "word count".

Thirteen studies using the Linguistic Inquiry Word Count (LIWC) program (Newman, Pennebaker, Berry, \& Richards, 2003) met the inclusion criteria. The initial statistical synopsis of these LIWC studies is presented below. The conference presentation will additionally include the meta-analysis of all other studies meeting the inclusion criteria $(\mathrm{k}=16)$ using different computer programs (e.g., General Architecture for Text Extraction (GATE), Agent99-Analyzer, CohMetrix).

\subsection{Independent Variables Coded}

(a) number of senders, (b) number of linguistic categories used, (c) medium used by senders to provide accounts, (d) type of and valence of the event, (e) senders' motivation, (f) senders' preparation, $(\mathrm{g})$ theory motivating the selection of categories, and (h) predictions for specific categories.

\subsection{Dependent Variables Coded}

(a) Effect sizes for each category in discriminating between truths and lies, (b) logistic regression or multiple discriminant analysis results for truths, lies, and overall classifications, and (c) reliability of each category.

\subsection{Effect Size Measure}

In order to compare the results from different studies, we computed the standardized mean difference as an effect size, which is referred to as Cohen's d (1988). Formula for computation of Cohen's $\mathrm{d}$ and for the entire meta-analytic procedure can be found in Cooper, Hedges, and Borenstein (2009), Hedges and Olkin (1985), or Lipsey and Wilson (2001). Cohen (1988) cautiously classified the effect size $d$ into three categories of magnitude, with $\mathrm{d}=.20$ defined as small, $\mathrm{d}=.50$ defined as medium and $\mathrm{d}=.80$ defined as large effect sizes. If a specific linguistic cue was more often used during deception than in a true story, $d$ becomes a negative sign. In case a linguistic cue occurred more often during a true than a deceptive story, $\mathrm{d}$ becomes a positive value.

\section{Results and Discussion}

\subsection{Descriptive Analyses}

Results of $k=13$ LIWC studies (from 9 sources; $k=5$ published and $k=4$ unpublished) revealed that most of the studies $(k=11)$ examined English transcripts, and two either Spanish or Dutch transcripts. In sum, 1143 transcripts were analyzed with a mean of 111 per study, which were given (handwritten or typed $(k=5)$, audiotaped or videotaped $(k=6)$ by 697 individuals. Senders' task was to lie or tell the truth about different topics, and in $38.46 \%$ of cases the story's valence was negative. Senders were slightly motivated in $60 \%$ of the studies, either receiving a small amount of money or a short verbal instruction.

Before analyzing the transcripts, they were corrected for errors (according to the manual) in 9 studies, whereas the remaining 4 did not report on that. From 68 default linguistic LIWCcategories, on average, 42 dimensions $(k=10)$ were analyzed at times with respect to a theoretical background (e.g., cognitive or emotional approaches, Reality Monitoring). Other categories were excluded due to a low base rate or due to nonsignificant findings. 


\subsection{Meta-analytic results}

Effect sizes with negative signs indicate that liars used the linguistic categories at a higher rate. At this point, 15 categories were chosen with at least $k=5$ each. Liars tend to use more words expressing negative emotions $(d=-0.111, p=$ $.041, k=13$, ) and positive emotions $(d=-0.201$, $p=.030, k=5$ ), more emotional words (Figure 1, $d=-0.187, p=.046, k=5$ ), more motion verbs $(d=-0.141, p=.011, k=12)$, and more negation words $(d=-0.188, p=.010, k=4)$.

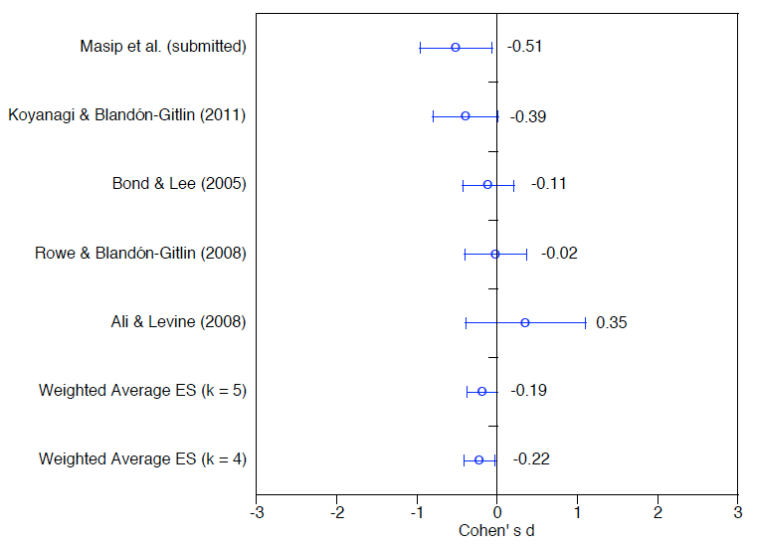

Figure 1. Distribution of Individual Effect Sizes for Emotion Words.

In contrast, truth-tellers make more use of selfreferences than liars $(d=0.123, p=.044, k=10)$, other-references $(d=0.138, p=.019, k=10)$, exclusive words (Figure 2, $d=0.360, p=.000, k$ $=12)$, slightly more tentative words $(d=0.172, p$ $=.071, k=4)$ or time-related words $(d=0.177, p$ $=.057, k=5)$ than liars.

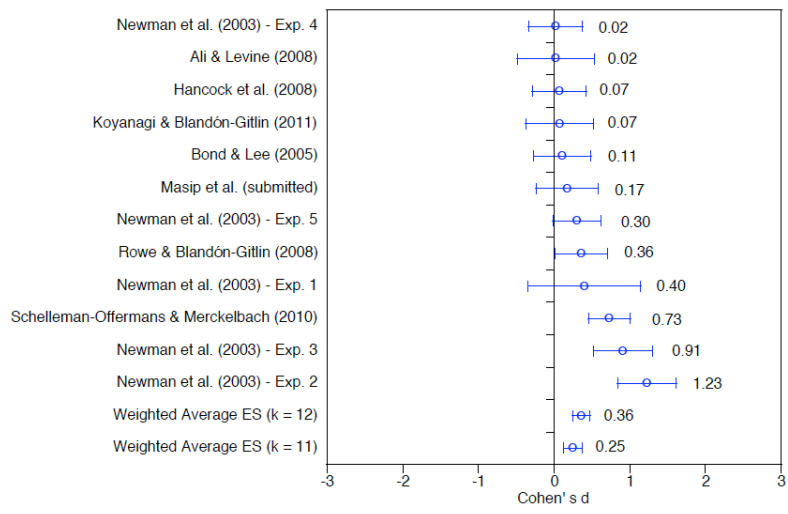

Figure 2. Distribution of Individual Effect Sizes for Exclusive Words.

No significant differences between liars and truth-tellers emerged for word count (Figure 3), the use of sensual and perceptual words, cognitive mechanisms or certainty words.

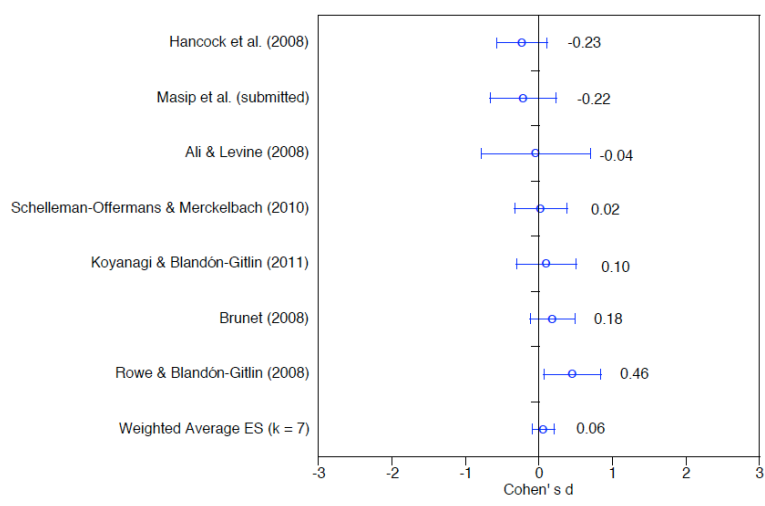

Figure 3. Distribution of Individual Effect Sizes for Word Count.

Although we found significant differences for some categories, we have to be aware of their general small magnitude (mean of all unweighted and absolute $d \mathrm{~s}=0.122$, mean of all weighted and absolute $d \mathrm{~s}=0.137$ ) and the small numbers of studies within each meta-analysis.

While some linguistic categories included in LIWC studies do not appear to have empirical precedence (e.g., motion verbs), others do have support from cognitive and emotional theoretical approaches (Bond \& Lee, 2005). It has been proposed that truth-tellers make more selfreferences because they are more likely than liars to associate themselves with the communication. Similarly, whereas truth-tellers are believed to use more exclusive words, signaling more complex explanations of what occurred, liars are believed to engage less in such explanations. Time, affect, space-related, and sensory words are features in accounts based on experienced events as predicted by the Reality Monitoring framework (Mitchell \& Johnson, 2000; Sporer, 2004). Negative emotion words are predicted to be higher in deceptive than true statements due to guilt or anxiety associated with the act of deception (Vrij, 2008). These predictions were partially supported by the current meta-analysis. Further meta-analyses with other computer programs (e.g., Fuller, Biros, Burgoon, Adkins, \& Twitchell, 2006; Humpherys, Moffitt, Burns, Burgoon, \& Felix, 2011; Zhou, Burgoon, Nunamaker, \& Twitchell, 2004) and theoretically driven moderator analyses (e.g., difference between children and adults, the effect of senders' motivation or preparation) will elucidate the linguistic pattern of truth-telling versus lying under specific conditions. 


\section{References}

References marked with an asterisk are included in the meta-analysis.

*Ali, M. \& Levine, T. (2008). The language of truthful and deceptive denials and confessions. Communication Reports, 21, 82-91.

Bond, C. F., \& DePaulo, B. M. (2006). Accuracy of deception judgments. Personality and Social Psychology Review, 10, 214-234.

*Bond, G. D., \& Lee, A. Y. (2005). Language of lies in prison: Linguistic classification of prisoners' truthful and deceptive natural language. Applied Cognitive Psychology, 19, 313-329.

*Brunet, M. K. (2009). Why bullying victims are not believed: Differentiating between children's true and fabricated reports of stressful and nonstressful events (Unpublished master's thesis). University of Toronto, Toronto.

Cohen, J. (1988). Statistical power analysis for the behavioural sciences (2nd ed.). Hillsdale, NJ: Erlbaum.

Cooper, H., Hedges, L. V., \& Valentine, J. C. (Eds.) (2009). The handbook of research synthesis and meta-analysis (2nd ed.). New York: Russell Sage Foundation.

DePaulo, B. M., Lindsay, J. J., Malone, B. E., Muhlenbruck, L., Charlton, K., \& Cooper, H. (2003). Cues to deception. Psychological Bulletin, 129, 74-118.

*Fuller, C., Biros, D. P., Burgoon, J. K., Adkins, M. Twitchell, D. P. (2006). An analysis of text-based deception detection tools, Proceedings of the 12th Americas Conference on Information Systems, Acapulco, Mexico.

*Hancock, J. T., Curry, L. E., Goorha, S., \& Woodworth, M. (2008). On lying and being lied to: A linguistic analysis of deception in computermediated communication. Discourse Processes, 45, $1-23$.

Hauch, V., Sporer, S. L., Michael, S. W., \& Meissner, C. A. (2010, June). Does training improve detection of deception? A meta-analysis. Paper presented at the 20th Conference of the European Association of Psychology and Law, Gothenburg, Sweden.

Hedges, L. V., \& Olkin, I. (1985). Statistical methods for meta-analysis. New York: Academic Press.

*Humpherys, S. L., Moffitt, K. C., Burns, M. B., Burgoon, J. K., Felix, W. F. (2011). Identification of fraudulent financial statements using linguistic credibility analysis. Decision Support Systems, 50, 585-594.
*Koyanagi, J. \& Blandón-Gitlin, I. (2011, March). Analysis of Children's Deception with the Linguistic Inquiry and Word Count Approach. Poster session presented at the 4th International Congress on Psychology and Law / 2011 Annual Meeting of the American Psychology-Law Society, Miami, Florida.

Levine, T. R., Park, H. S., \& McCornack, S. A. (1999). Accuracy in detecting truths and lies: Documenting the "veracity effect". Communication Monographs, 66, 125-144.

Lipsey, M. W., \& Wilson, D. B. (2001). Practical meta-analysis. Thousand Oaks: Sage Publications.

*Masip, J., Bethencourt, M., Lucas, G., Sánchez-San Segundo, M., \& Herrero, C. (2011). Deception detection from written accounts. Scandinavian Journal of Psychology.

Meissner, C. A. \& Kassin, S. M. (2002). "He's guilty!": Investigator bias in judgments of truth and deception. Law and Human Behavior, 26, 469-480.

Mitchell, K. J., \& Johnson, M. K. (2000). Source monitoring: Attributing mental experiences. In E. Tulving, \& F. I. M. Craik (Eds.), The Oxford Handbook of Memory (pp. 179-195). New York: Oxford University Press.

*Newman, M. L., Pennebaker, J. W., Berry, D. S., \& Richards, J. M. (2003). Lying words: Predicting deception from linguistic styles. Personality and Social Psychology Bulletin, 29, 665-675.

*Rowe, K. \& Blandón-Gitlin, I. (2008, March). Discriminating true, suggested, and fabricated statements with the Linguistic Inquiry and Word Count approach. Poster session presented at the Annual Meeting of the American Psychology-Law Society, Jacksonville, Florida.

*Schelleman-Offermans, K., \& Merckelbach, H. (2010). Fantasy proneness as a confounder of verbal lie detection tools. Journal of Investigative Psychology and Offender Profiling, 7, 247-260.

Sporer, S. L. (2004). Reality monitoring and the detection of deception. In P.-A. Granhag \& L. Stromwall (Eds.), Deception detection in forensic contexts (pp. 64-102). Cambridge University Press.

Vrij, A. (2008). Detecting lies and deceit: Pitfalls and opportunities. Chichester, England: Wiley.

*Zhou, L., Burgoon, J. K., Nunamaker, J. F., \& Twitchell, D. (2004). Automating linguistics-based cues for detecting deception in text-based asynchronous computer-mediated communication. Group Decision and Negotiation, 13, 81-106. 